\title{
Individual and relational risk factors for the development of eating disorders in adolescent aesthetic athletes and general adolescents
}

\author{
Rita Francisco $\cdot$ Isabel Narciso • Madalena Alarcão
}

Received: 4 December 2012 / Accepted: 18 July 2013/Published online: 13 August 2013

(C) Springer International Publishing Switzerland 2013

\begin{abstract}
This study compared potential risk and protective factors, levels of disordered eating (DE), and their relationship among young aesthetic athletes (elite and nonelite) and controls $(N=725 ; 62.5 \%$ females; mean age $=15.3, \mathrm{SD}=2.1)$. The participants completed selfreport measures (McKnight Risk Factor Survey-IV, Contour Drawing Rating Scale and Eating Disorder Examination-Questionnaire). Female elite athletes showed a greater risk of developing eating disorders than non-elite athletes and controls, with no difference between the three groups of males. Multiple group analyses revealed important differences in DE predictors. Although social pressure is the strongest DE predictor in non-elite athletes and controls, in elite athletes, the strongest DE predictor is body image dissatisfaction. Parental influences, rather than self-esteem, are predictors of DE in elite athletes, unlike the other two groups. These results show that the risk and protective factors involved in the development of DE are not universally valid. The results highlight the importance of studying specific characteristics associated with DE in
\end{abstract}

R. Francisco $\cdot$ I. Narciso

Faculty of Psychology, University of Lisbon, Lisbon, Portugal

R. Francisco $(\bowtie) \cdot$ I. Narciso

Faculdade de Psicologia, Alameda da Universidade, 1649-013 Lisbon, Portugal

e-mail: rmfrancisco@fp.ul.pt

M. Alarcão

Faculty of Psychology and Education Sciences,

University of Coimbra, Coimbra, Portugal

M. Alarcão

Faculdade de Psicologia e de Ciências da Educação, Rua do Colégio Novo, Apartado 6153, 3001-802 Coimbra, Portugal aesthetic athletes. Some implications for ED risk assessment and prevention are discussed.

Keywords Aesthetic athletes - Adolescents .

Disordered eating $\cdot$ Risk factors · Protective factors

\section{Introduction}

Numerous individual, familial, and sociocultural risk and protective factors have been identified (e.g., [1]), demonstrating the multifactorial nature of eating disorders (ED). However, the way in which these factors interact remains unclear, making it difficult to establish the etiology of ED or to design effective preventive or therapeutic approaches [2].

Thirty years ago, Garner and Garfinkel [3] drew attention to the fact that involvement in activities that demand more attention and control over body image may be considered an additional risk for the development of ED. Two meta-analyses conducted on studies of athletes in various sports showed a greater prevalence of ED in comparison to the general population, especially among specific sports with high levels of competition [4, 5]. In fact, a relationship has been identified between aesthetic sports, which emphasize thinness and associated improvements in performance, such as dance, gymnastics or figure skating, and higher levels of clinical and sub-clinical ED syndromes in both sexes (e.g., [6, 7]). In contrast, Smolak et al. [5] demonstrated that non-elite athletes, especially those in high school, had a reduced risk of eating problems compared to controls. Therefore, the main purpose of this study is to extend research that analyses risk and protective factors associated with the development of ED in aesthetic sports athletes at different levels of competition and to 
understand the similarities and differences between these athletes and in relation to the general adolescent population.

Although females are more susceptible to ED because of their higher concerns about weight and body image, recent studies have suggested that such concerns are increasing among males [8]. Among athletes, females appear to be at greater risk, although few studies have included male aesthetic athletes. According to a meta-analysis of studies including male athletes [4], the difference between male athletes and male controls is greater than the difference between female athletes and female controls. Various studies have found controversial results related to ED among male athletes and controls (e.g., [9, 10]).

Various individual characteristics are associated with ED, such as perfectionism, low self-esteem and body dissatisfaction (e.g., [11]). Unlike perfectionism, which is a personality trait and therefore is a relatively stable characteristic throughout life, low self-esteem and body dissatisfaction seem to emerge with greater intensity during puberty and develop concurrently in young people of both genders. Although these two issues are similar, they do not appear to cause one another (e.g., [12]). A recent study of female collegiate athletes showed that low levels of selfesteem, but not high levels of perfectionism, were associated with higher levels of disordered eating [13]. Some studies show a clear correlation between low body image satisfaction and ED symptoms among athletes (e.g., [14, 15]), even considering body image satisfaction a mediating variable for the social influence for thinness, anxiety about athletic performance, and negative appraisals of athletic achievement [16]. However, other studies report the absence of this correlation. For example, athletes showed higher levels of ED than non-athletes but similar incidences of body dissatisfaction (e.g., [17]).

In relation to socio-relational factors, society is seen as a vehicle for somewhat unrealistic and unhealthy aesthetic ideals, especially for females, whose comparisons of their own bodies with those depicted in the media contribute to concerns about image and weight [18]. However, it is possible that the messages in the media only become a problem if and when they are reinforced by the socializing agents closest to the adolescents, such as peers and family, or other significant role models, such as coaches (for athletes). Countless studies have shown a significant impact of appearance-related teasing and criticism on weight concerns, body dissatisfaction, self-esteem, depressive symptoms and ED, both in the general population (e.g., [19, 20]), and among athletes (e.g., [21]). Although significantly fewer studies have considered male participants, very similar patterns of influence have been found in adolescents of both sexes (e.g., [12]). However, a high level of social support has been considered an important protective factor from ED (e.g., [22]) despite the lack of studies of athletes showing this variable as a possible predictor of ED.

A large amount of the literature establishes body image dissatisfaction (BID) and low self-esteem as strong predictors of ED in athletes and the general population. This study considers these issues potential (partial or full) mediators of the influence of other factors (female, high BMI, perfectionism, pressure for thinness, low social support) on disordered eating. Furthermore, these issues may also be mediators of other outcomes, such as substance abuse and depressive-anxiety symptomatology, which are believed to be risk factors for ED development, through frequent comorbidity (e.g., [23]).

The objectives of this study are to compare female and male elite athletes, non-elite athletes and controls in terms of (a) potential risk/protective factors for the development of ED, (b) levels of disordered eating, and (c) the relationship between risk/protective factors and disordered eating. Because of the exploratory nature of the study, no hypotheses are provided.

\section{Method}

\section{Participants}

The participants were 725 adolescents (453 girls, 272 boys; $M=15.34$ years, $S D=2.12$ ), 245 adolescents who were aesthetic athletes (54.3\% elite) and 480 adolescents who did no aesthetic sports (control group). Elite athletes are internationally competitive gymnasts and professional dance students. Non-elite athletes are gymnasts from lower levels of competition and recreational dancers.

The participants lived in different areas of Portugal, including the Lisbon Metropolitan Area (46.9\%), Center $(43.6 \%)$, North $(6.9 \%)$, South $(2.3 \%)$ and Madeira Islands $(0.3 \%)$.

\section{Measures}

\section{McKnight Risk Factor Survey-IV (MRFS-IV)}

The MRFS-IV is a self-report questionnaire created to evaluate the potential risk factors in the development of ED in female adolescents [24]. A Portuguese version, which includes both male and female versions [25], was used. The nine factors (consisting of 62 items rated on a 5-point Likert scale) present adequate internal consistency: concern with thinness $(\alpha=0.96)$, self-esteem $(\alpha=0.87)$, social pressure $(\alpha=0.91)$, overeating $(\alpha=0.82)$, substance use and compensatory methods $(\alpha=0.65)$, social support $(\alpha=0.88)$, parental influences $(\alpha=0.72)$, 
anxiety-depression symptomatology $(\alpha=0.78)$ and perfectionism $(\alpha=0.71)$.

\section{Contour Drawing Rating Scale (CDRS)}

The CDRS, which consists of a sequence of nine silhouettes ordered from the thinnest to the largest, assesses BID by requiring participants to indicate which silhouette best represents their current and ideal body sizes [26]. The current-ideal discrepancy indicates BID. The Portuguese version used in this study [27] presents good reliability on the test-retest index $(r=0.91)$ and construct validity (correlations of the silhouette representing current body size with weight $r=0.65$ and with BMI $r=0.72$ ).

\section{Eating Disorder Examination-Questionnaire (EDE-Q)}

The Portuguese version of the 5th edition of the EDE-Q [29] was used to assess participants along a continuum of disordered eating. It consists of a self-report with 28 items rated on a 7-point Likert scale and grouped into four subscales (restraint, eating concern, shape concern, and weight concern). The average result represents a global score [28]. For this sample, internal consistency for the global score was $\alpha=0.92$, slightly lower than for the Portuguese population $(\alpha=0.95$; [29]).

\section{Procedure}

The coordinators of 16 national gymnastics clubs, 10 professional and recreational dance schools, and 8 secondary schools were approached. All of them agreed to cooperate, and parental and adolescent informed consent was obtained. The data collection (approximately $40 \mathrm{~min}$ ) was scheduled around training/classes and occurred in groups in the presence of the researcher. Permission to conduct the study was provided by the Ministries of Science (FCT) and Education.

\section{Statistical analyses}

Statistical analysis was conducted using SPSS 17.0. The Kruskal-Wallis test was used to evaluate the mean differences, and the Mann-Whitney test was used as a post hoc procedure with the application of the Bonferroni correction. A Chi-square test was used to evaluate the differences in the clinically disordered eating indicator. Structural equation modeling (SEM), particularly multiple group analysis with the AMOS software, was used to test an initial global model of adolescents' disordered eating and to compare competing structural models [30]. Because most studies indicate more similarities than differences between risk factors for the development of ED among girls and boys [31], both sexes were included in the same models. In all analyses, the maximum likelihood estimation method and the covariance matrix were used. The overall goodness of fit was based on the combination of several fit indices [32]. Models were compared based on Chi-square difference tests and on other fit indices, including the standardized root mean square (SRMR), incremental index of fit (IFI), and root mean square error of approximation (RMSEA). To assess whether indirect effects were statistically significant, bias-corrected (BC) bootstrap $95 \%$ confidence intervals (CIs) with a 5,000 bootstrap sample procedure were used [33].

\section{Results}

Descriptive statistics and differences between groups

Table 1 shows the descriptive statistics of all variables and the mean differences for the three groups (elite athletes, non-elite athletes and controls) of males and females.

\section{Risk and protective factors}

Among females, the mean differences were statistically significant for the majority of the variables with the exception of self-esteem, social support, parental influences, and perfectionism. The BMI of elite and non-elite athletes was similar, but the controls presented a significantly higher BMI than the elite athletes $(U=9,525.00, p<0.001)$ or the nonelite athletes $(U=10,415.50, p<0.01)$. The elite athletes were significantly more dissatisfied with their body image than the non-elite athletes were $(U=3,997.00, p<0.01)$, idealizing a thinner body image than their current one. However, they showed levels of BID that were similar to the control group, which did not differ significantly from nonelite athletes. The elite athletes showed greater concern with thinness than the non-elite athletes $(U=3,049.00$, $p<0.001)$ and controls $(U=9,220.00, p<0.01)$. The elite athletes also scored higher on social pressure compared with the non-elite athletes $(U=3,768.50, p<0.01)$, but they did not score higher than the controls, who also did not differ from the non-elite athletes. The non-elite athletes showed significantly lower levels of overeating than the elite athletes ( $U=3,888.00, p<0.01)$ and the controls $(U=10,480.00$, $p<0.01)$. Regarding substance use and compensatory methods, elite and non-elite athletes did not differ, but these groups differed from the control group, which showed significantly higher levels $(U=10,409.50, \quad p<0.01$; $U=8,518.50, p<0.001$, respectively). Finally, the controls showed significantly higher levels of anxiety-depression symptomatology than the non-elite athletes $(U=9,944.50, p<0.01)$ but similar levels as the elite athletes, who did not differ from the non-elite athletes. 
Table 1 Descriptive statistics and results of comparison means $(N=725)$

\begin{tabular}{|c|c|c|c|c|c|c|c|c|c|c|}
\hline & \multicolumn{5}{|l|}{ Girls } & \multicolumn{5}{|l|}{ Boys } \\
\hline & $\begin{array}{l}\text { Elite } \\
(N=101)\end{array}$ & $\begin{array}{l}\text { Non-elite } \\
(N=99)\end{array}$ & $\begin{array}{l}\text { Control } \\
(N=253)\end{array}$ & $H(2)$ & $p$ & $\begin{array}{l}\text { Elite } \\
(N=30)\end{array}$ & $\begin{array}{l}\text { Non-elite } \\
(N=15)\end{array}$ & $\begin{array}{l}\text { Control } \\
(N=227)\end{array}$ & $H(2)$ & $p$ \\
\hline BMI & $19.16(2.15)$ & $19.52(2.55)$ & $20.36(2.88)$ & 14.17 & $<0.01$ & $20.43(2.71)$ & $21.43(3.29)$ & $21.10(3.30)$ & 0.82 & ns \\
\hline $\begin{array}{l}\text { Concern with } \\
\text { thinness }\end{array}$ & $2.70(1.06)$ & $2.02(0.94)$ & $2.20(0.98)$ & 24.53 & $<0.001$ & $1.63(0.75)$ & $1.61(0.62)$ & $1.58(0.76)$ & 0.87 & ns \\
\hline Self-esteem & $3.38(0.76)$ & $3.56(0.69)$ & $3.36(0.80)$ & 5.25 & ns & $3.68(0.71)$ & $3.86(0.55)$ & $3.70(0.74)$ & 0.41 & ns \\
\hline Social pressure & $1.43(0.59)$ & $1.26(0.41)$ & $1.41(0.60)$ & 8.43 & $<0.05$ & $1.26(0.47)$ & $1.02(0.05)$ & $1.24(0.50)$ & 7.51 & .05 \\
\hline Overeating & $2.00(0.85)$ & $1.66(0.67)$ & $1.89(0.79)$ & 8.52 & $<0.05$ & $1.59(0.60)$ & $1.49(0.52)$ & $1.61(0.66)$ & 0.23 & ns \\
\hline $\begin{array}{l}\text { Sub. use/comp. } \\
\text { methods }\end{array}$ & $1.26(0.39)$ & $1.18(0.36)$ & $1.37(0.45)$ & 25.42 & $<0.001$ & $1.24(0.32)$ & $1.18(0.21)$ & $1.27(0.32)$ & 0.60 & ns \\
\hline Social support & $4.07(1.01)$ & $4.09(0.97)$ & $4.11(0.95)$ & 0.23 & ns & $3.64(0.95)$ & $3.76(1.07)$ & $3.48(1.19)$ & 1.29 & ns \\
\hline Parental influences & $1.42(0.67)$ & $1.27(0.48)$ & $1.35(0.58)$ & 2.95 & ns & $1.27(0.44)$ & $1.12(0.21)$ & $1.25(0.51)$ & 1.22 & ns \\
\hline $\begin{array}{l}\text { Anxiety-depression } \\
\text { symptomatology }\end{array}$ & $2.15(0.54)$ & $2.07(0.58)$ & $2.29(0.62)$ & 10.90 & $<0.01$ & $2.04(0.48)$ & $1.79(0.39)$ & $1.89(0.54)$ & 3.50 & ns \\
\hline Perfectionism & $2.52(0.92)$ & $2.71(0.90)$ & $2.59(0.92)$ & 1.56 & ns & $2.83(0.86)$ & $2.97(0.84)$ & $2.56(0.96)$ & 5.47 & ns \\
\hline BID & $-0.88(1.21)$ & $-0.51(1.19)$ & $-0.81(1.31)$ & 6.68 & $<0.05$ & $-0.11(0.83)$ & $0.27(0.96)$ & $-0.17(1.09)$ & 1.41 & ns \\
\hline EDE-Q Global Score & $1.69(1.32)$ & $1.01(1.11)$ & $1.22(1.25)$ & 19.22 & $<0.001$ & $0.56(0.84)$ & $0.34(0.26)$ & $0.52(0.77)$ & 0.95 & ns \\
\hline
\end{tabular}

Males only differed significantly in social pressure. Non-elite athletes reported less social pressure to be thin than did the controls $(U=1,032.00, p<0.01)$. The elite athletes did not differ from their non-elite peers or from the controls.

\section{Disordered eating (DE)}

Female elite athletes showed a significantly higher level of DE than non-elite athletes $(U=3,199.50, p<0.001)$ or controls $(U=9,605.00, p<0.001)$, with no significant difference between non-elite athletes and controls. There were no statistically significant differences among males.

According to the EDE-Q cut-off for the Portuguese population $(\geq 3.5 ; 29), 34 \%$ of the female elite athletes, $15.3 \%$ of the non-elite athletes and $21.7 \%$ of the controls showed a clinical level of $\mathrm{DE}\left[\chi^{2}(2)=10.32, p<0.01\right]$. The DE level is not independent of the group to which an adolescent belongs: elite athletes reveal significantly higher levels of $\mathrm{DE}$ than non-elite athletes $\left[\chi^{2}(1)=9.29\right.$, $p<0.01]$ and controls $\left[\chi^{2}(1)=5.71, p<0.05\right]$. Non-elite athletes and controls do not differ from one another. Among males, there were no cases in non-elite athletes, and only $6.7 \%$ of elite athletes and $6.2 \%$ of controls showed DE at a clinical level, independent of belonging to a specific group $\left[\chi^{2}(2)=1.00, p=0.76\right]$.

Predictors of disordered eating

To create a parsimonious model of DE development in adolescents, the only variables chosen were those that showed at least moderate correlations $(r>0.40)$ with the EDE-Q global score, considering the general indicator of $\mathrm{DE}^{1}$ in the three groups (sex, BMI, concern with thinness, social pressure, parental influences, self-esteem, BID, overeating, and anxiety-depression symptomatology). The concern with thinness factor of the MRFS-IV showed an excessively high correlation with the EDE-Q global score ( $r=0.85$ total sample, $r=0.86$ control group, $r=0.82$ elite and non-elite athlete groups), which may complicate the creation of the model [34]. At the same time, the content analysis of the items that constitute these two latent variables were similar (feeling fat, dieting, etc.). Therefore, we decided to eliminate the first variable because EDE-Q is better established in the literature as a good measure of DE.

To test the influence of the selected variables on adolescents' DE, we used a model-generating application of SEM [34]. Given the problems caused by the estimation of all observed items and latent variables (insufficient power and under-identification; [35]), we assumed the scale and latent variables to be identical. An initial model was tested with the total sample and showed a good fit to the data $\left[\chi^{2}(11)=42.86, p<0.001 ;\right.$ SRMR $=0.04 ;$ IFI $=0.99$; RMSEA $=0.06]$. The non-significant relationships (structural paths from parental influences to BID and from BID to overeating and anxiety-depression symptomatology) were withdrawn from the subsequent analyses to achieve

\footnotetext{
${ }^{1}$ Because of their excessive length, the correlation tables of the variables of the three study groups are not presented but are available upon request.
} 
results that were both theoretically sound and empirically supported.

The groups were compared using multiple group analyses. The baseline model demonstrated a good fit to the data $\left[\chi^{2}(30)=78.37, p<0.001 ;\right.$ SRMR $=0.04 ;$ IFI $=0.98$; RMSEA $=0.05]$. We tested for equality constraints for each structural relationship and found significant differences by group for all of them. We then performed a series of two-group analyses with equality constraints (elite-nonelite athletes, elite athletes-controls and non-elite athletescontrols). Only the structural path from social pressure to DE proved to be non-significant for the comparisons of nonelite athletes-controls $\left[\Delta \chi^{2}(2)=5.74, p=0.057\right]$ and elite-non-elite athletes $\left[\Delta \chi^{2}(2)=3.68, p=0.159\right]$.

After withdrawing the non-significant structural paths for each group, the three models produced acceptable fits, especially Model C [control group; $\chi^{2}(12)=32.30$, $p<0.01 ;$ SRMR $=0.04 ;$ IFI $=0.99 ;$ RMSEA $=0.06]$, followed by Model B [non-elite athletes; $\chi^{2}(17)=28.28$, $p<0.05$; $\mathrm{SRMR}=0.07$; $\mathrm{IFI}=0.97 ; \mathrm{RMSEA}=0.08$ ]. Model A (elite athletes) produced the worst fit $\left[\chi^{2}(20)=44.91, \quad p<.01 ; \quad\right.$ SRMR $=0.12 ; \quad$ IFI $=0.94 ;$ $\mathrm{RMSEA}=0.10]$, most likely revealing the existence of additional risk factors in sports contexts that are not found in the general population. Figure 1 represents the three models with the standardized regression weights and significance levels for each structural path. The amount of variance explained for $\mathrm{DE}$ is $64.6 \%$ for the controls, $64.7 \%$ for the non-elite athletes, and $63.7 \%$ for the elite athletes.

All path coefficients for the indirect effects were significant and in the expected directions (Table 2).

\section{Discussion}

In this study, we aimed to compare female and male elite athletes, non-elite athletes and controls in terms of (a) potential risk/protective factors for the development of eating disorders, (b) levels of disordered eating, and (c) the relationship between risk/protective factors and disordered eating.

Differences between elite athletes, non-elite athletes and controls are especially prevalent among females. Males are much more homogeneous in terms of potential risk/protective factors and the level of DE.

In fact, only the perception of social pressure for thinness is significantly different between males of the control group and non-elite athletes, with the latter showing lower levels of pressure. Interestingly, the pressure felt by elite athletes and controls does not differ significantly, and the same pattern of differences for this variable occurs with females. The fact that non-elite athletes practice an aesthetic sport may lead to the development of a body closer to the social ideal, making these athletes less likely to be victims of critical comments. In contrast, the similar level of pressure for thinness felt by elite athletes and controls may be because, although they have a lower BMI than controls, these athletes (especially females) are targets of additional pressure in the context of their sport to present a thin body image appropriate to the sport [17] in addition to the pressures to which most adolescents are already subject.

Interestingly, in males, the differences in social pressure are not accompanied by differences in BID or DE. This finding does not agree with studies in which male non-athletes showed higher BID than athletes (e.g., [36]). However, some differences between the three groups of males may not have been detected due to the restricted sample of both elite and non-elite athletes, making it imperative that future studies include larger samples of male athletes.

Regarding the hypothesized protective factors (social support and self-esteem), neither showed significant differences among elite athletes, non-elite athletes and controls for males or females. Because of the weak correlation with DE, social support does not appear to be a protective factor in the development of eating disorders for adolescents from the general population [22] or athletes. Regarding self-esteem, the absence of significant differences between athletes and controls is unexpected in light of studies that indicate higher self-esteem in the former in comparison to the latter (e.g., [37]) as well as in elite athletes compared with non-elite athletes and non-athletes [38]. The authors associate these differences with the greater physical competence shown by elite athletes, which may not contribute in the same way to the global selfesteem of the athletes in our sample. However, unlike social support, self-esteem showed significant correlations with the DE indicator and is therefore understood to be a protective factor.

The absence of significant differences in perfectionism supports the previous literature because there are contradictory results in a number of studies. Higher levels of perfectionism have been found in female adolescent dancers compared to non-dancers [6], but other studies have not found these differences [13]. Perfectionism does not seem to be a characteristic of adolescents engaged in elite sport activities, as suggested by Thomas et al. [39].

Being a non-elite athlete seems to protect female adolescents from developing depressive and/or anxious symptomatology; the results of this variable are significantly lower than those of the elite athletes and controls. This result is consistent with studies that show that collegiate athletes have lower levels of depressive symptoms than non-athletes, suggesting that participation in sports positively influences mental health outcomes [37]. 
Fig. 1 Final structural models of adolescents' disordered eating

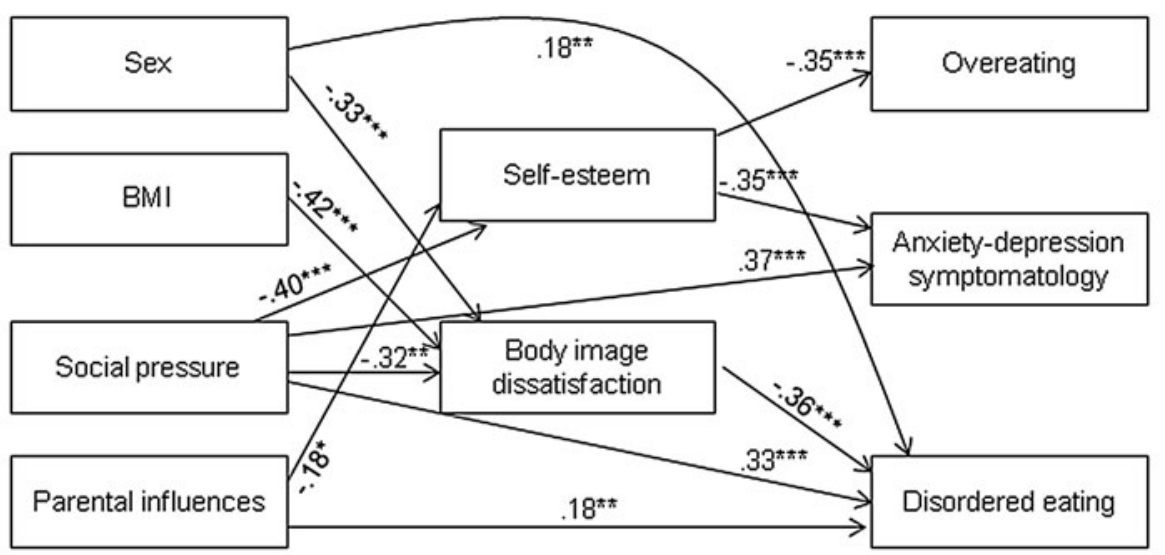

Model A: Final structural model of elite athletes' disordered eating.

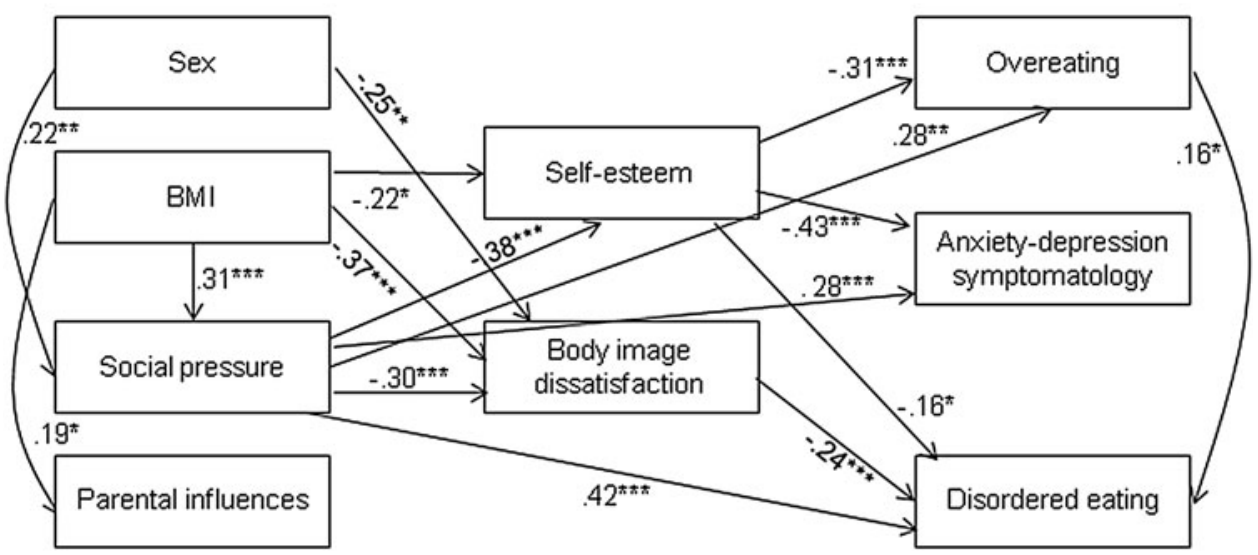

Model B: Final structural model of non-elite athletes' disordered eating.

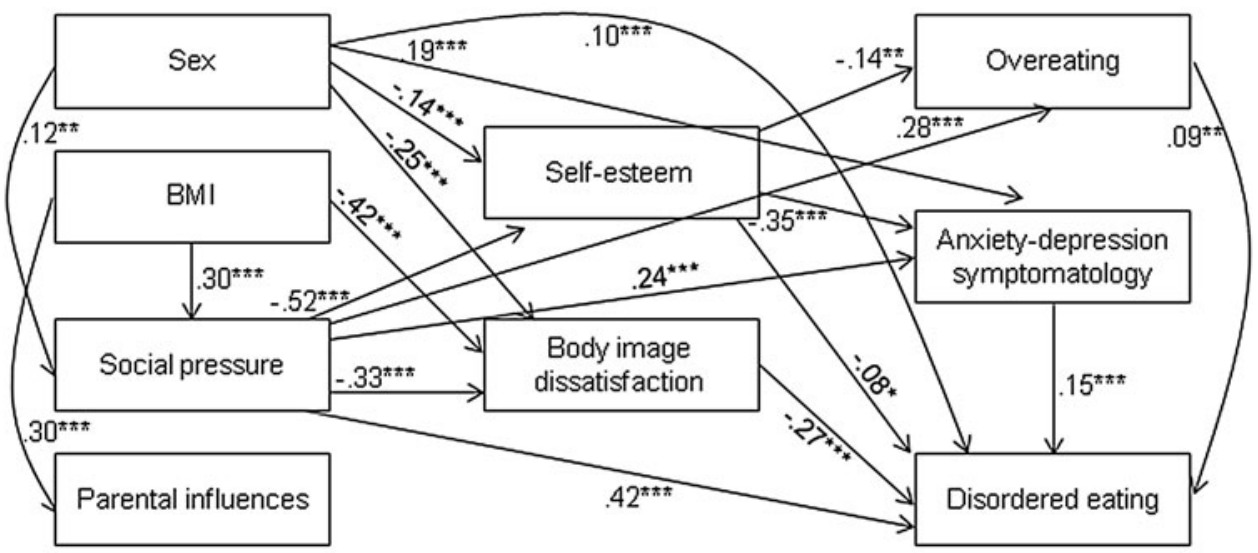

Model C: Final structural model of controls' disordered eating.

However, as demonstrated by SEM, anxiety-depression symptomatology is not a predictor of $\mathrm{DE}$ in any group of athletes, unlike the control group. Therefore, this cannot be considered an indicator of lower risk of DE for non-elite athletes. However, lower indices of overeating and, especially, the higher body image satisfaction of non-elite athletes may indicate a reduced risk of DE compared with elite athletes and controls.
Female elite athletes showed significantly higher levels of concern with thinness and DE in comparison to the other two groups of females. This result is corroborated by the significantly higher percentage of cases identified by the EDE-Q cut-off. In all of these indicators, non-elite athletes and controls did not differ from one another, reinforcing the idea that the elite context of aesthetic sports creates a higher risk for adolescent females to develop eating 


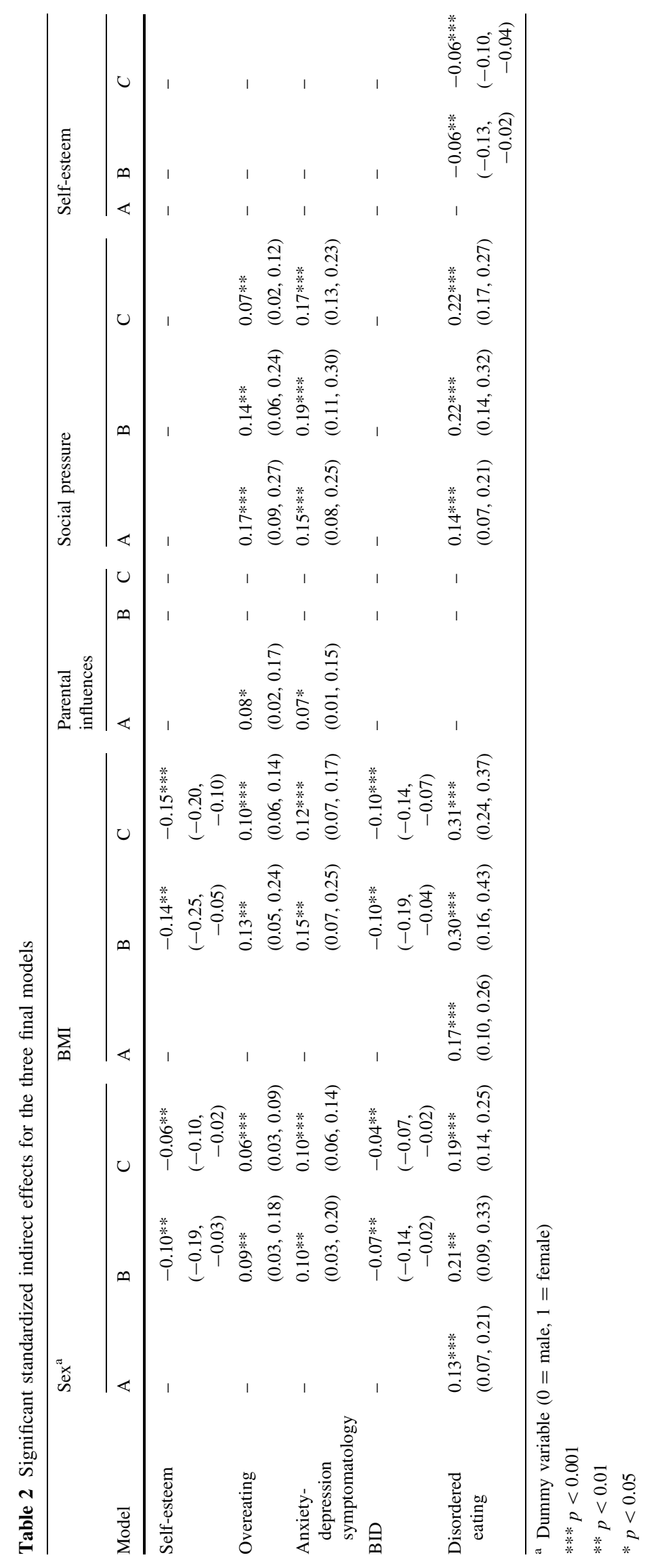


disorders (e.g., [7, 40]). However, unlike other authors [5, 10], we cannot affirm that non-elite athletes present a lower risk than non-athletes despite their lower BID; they seem to present a similar risk. Rosendahl et al. [10] recently found a significantly higher frequency of $\mathrm{DE}$ in both male and female elite athletes compared to non-elite athletes. This finding is partially corroborated by our results because this pattern is only noticeable for females. In our study, males showed DE regardless of their context, suggesting that males who participate in aesthetic sports at any level do not present a lower risk of eating disorders than non-athletes.

Multiple group analyses showed important differences in the way that different risk/protective factors predict DE in elite athletes, non-elite athletes and the general adolescent population. Torstveit et al. [41] suggested that different indicators of DE behaviors would predict clinical eating disorders among leanness/nonleanness athletes and controls. Our study also shows that the risk/protective factors involved in the development of DE are not universally valid. As suggested by the comparisons of the mean of risk factors and DE, the final structural models show similarities between non-elite athletes and controls and divergences between these two groups and elite athletes. Specifically, it is interesting to note that social pressure has an influence (both directly and indirectly) that is significantly higher on non-elite athletes and controls than on elite athletes' DE. This pressure, unlike the pressure on elite athletes, is influenced by sex and BMI. In turn, parental influences are predictors of low self-esteem and DE only among elite athletes. This is a new and very important finding because primacy is often given to the impact of critical comments by coaches (e.g., [21]). Future studies should explore the impact of other family variables on elite athletes' DE.

To synthesize the role of the mediating variables, it is worth highlighting some differences in the three groups. BID, which is predicted by the same risk factors in all three groups (sex, BMI and social pressure), corresponds to the strongest predictor of DE in elite athletes but not for nonelite athletes or controls. For the latter two groups, social pressure has a more relevant role. This result makes it essential to deepen the study of BID among elite athletes and the context that may influence it, both positively and negatively, in an effort to design effective preventive actions. Self-esteem, which is considered a relevant protective factor in this model, was not a predictor of DE in elite athletes, in contrast to the other two groups. This was an unexpected result, especially due to the frequent association between DE and low self-esteem in athletes of aesthetic sports (e.g., [14, 15]). Furthermore, social pressure (which is related to peer pressure, as mentioned) is the strongest predictor of self-esteem in the three groups. At the same time that peers take on a fundamental role in adolescence, physical self-esteem corresponds to one of the main components of adolescents' self-esteem [42] and depends on the comments adolescents receive about their physical appearance. Therefore, we believe that the lesser influence of peers' critical comments on athletes' selfesteem compared to the controls can be explained by the particular benefits to adolescents' self-concept from participation in a recreational/competitive sport [38], particularly the attribution of greater importance to other components of self-esteem.

This study has some limitations. Although it fulfills the minimum criteria to undertake structural equation models [34], the small number of male athletes may have skewed the results. Future studies should test the same models with different samples of male and female athletes to determine the generalizability of these findings. Furthermore, the cross-sectional design of this study does not permit causal inference. Finally, a clinical evaluation of adolescents who reported (or did not report) disordered eating is needed to examine the prevalence of true eating disorders, especially among athletes. Many athletes, particularly those from leanness sports, underreport disordered eating behaviors [41].

Acknowledgments The authors thank all of the adolescents and their teachers and coaches for their participation in the study. Funding for this study was provided by the $\mathrm{PhD}$ grant to the first author (SFRH/BD/27472/2006) by the Fundação para a Ciência e a Tecnologia (FCT), Portugal.

Conflict of interest The authors declare that they have no conflict of interest.

\section{References}

1. Keery H, van den Berg P, Thompson JK (2004) An evaluation of the Tripartite Influence Model of body dissatisfaction and eating disturbance with adolescent girls. Body Image 1:237-251

2. Striegel-Moore R, Bulik C (2007) Risk factors for eating disorders. Am Psychol 62:181-198

3. Garner DM, Garfinkel P (1980) Socio-cultural factors in the development of anorexia nervosa. Psychol Med 10:647-656

4. Hausenblas H, Carron A (1999) Eating disorder indices and athletes: an integration. J Sport Exerc Psychol 21:230-258

5. Smolak L, Murnen S, Ruble A (2000) Female athletes and eating problems: a meta-analysis. Int J Eat Disord 27:371-380

6. Anshel M (2004) Sources of disordered eating patterns between ballet dancers and non-dancers. J Sport Behav 27:115-133

7. Sundgot-Borgen J, Torstveit M (2004) Prevalence of eating disorders in elite athletes is higher than in the general population. Clin J Sport Med 14:25-32

8. Kjelsås E, Bjørnstrøm C, Götestam K (2004) Prevalence of eating disorders in female and male adolescents (14-15 years). Eat Behav 5:13-25

9. Petrie TA, Greenleaf C, Reel J et al (2008) Prevalence of eating disorders and disordered eating behaviors among male collegiate athletes. Psychol Men Masc 9:267-277 
10. Rosendahl J, Bormann B, Aschenbrenner K et al (2009) Dieting and disordered eating in German high school athletes and nonathletes. Scand J Med Sci Sport 19:731-739

11. Ghaderi A (2001) Review of risk factors for eating disorders: implications for primary prevention and cognitive behavioural therapy. Scand J Behav Ther 30:57-74

12. Phares V, Steinberg A, Thompson J (2004) Gender differences in peer and parental influences: body image disturbance, self-worth, and psychological functioning in preadolescent children. J Youth Adolesc 33:421-429

13. Petrie TA, Greenleaf C, Reel J et al (2009) Personality and psychological factors as predictors of disordered eating among female collegiate athletes. Eat Disord 17:302-321

14. Bettle N, Bettle O, Neumärker U et al (2001) Body image and self-esteem in adolescent ballet dancers. Percept Motor Skill 93:297-309

15. Petrie TA (1993) Disordered eating in female collegiate gymnasts: prevalence and personality/attitudinal correlates. J Sport Exerc Psychol 15:424-436

16. Williamson D, Netemeyer R, Jackman L et al (1995) Structural equation modeling of risk factors for the development of eating disorder symptoms in female athletes. Int $\mathrm{J}$ Eat Disord $17: 387-393$

17. de Bruin A, Oudejans R, Bakker F (2007) Dieting and body image in aesthetic sports: a comparison of Dutch female gymnasts and non-aesthetic sport participants. Psychol Sport Exerc 8:507-520

18. Field A, Camargo C, Taylor C et al (2001) Peer, parent, and media influences on the development of weight concerns and frequent dieting among preadolescent and adolescent girls and boys. Pediatrics 107:54-60

19. Benas J, Gibb B (2008) Weight-related teasing, dysfunctional cognitions, and symptoms of depression and eating disturbances. Cogn Ther Res 32:143-160

20. Vincent M, McCabe M (2000) Gender differences among adolescents in family, and peer influences on body dissatisfaction, weight loss, and binge eating behaviors. J Youth Adolesc 29:205-221

21. Muscat A, Long B (2008) Critical comments about body shape and weight: disordered eating of female athletes and sport participants. J Appl Sport Psychol 20:1-24

22. McVey G, Pepler D, Davis R et al (2002) Risk and protective factors associated with disordered eating during early adolescence. J Early Adolesc 22:75-95

23. O’Brien K, Vincent N (2003) Psychiatric comorbidity in anorexia and bulimia nervosa: nature, prevalence, and causal relationships. Clin Psychol Rev 23:57-74

24. McKnight Investigators (2003) Risk factors for the onset of eating disorders in adolescent girls: results of the McKnight longitudinal risk factor study. Am J Psychiatry 160:248-254

25. Francisco R, Alarcão M, Narciso I (2011) Avaliação de factores de risco de desenvolvimento de perturbações alimentares:
Desenvolvimento e estudos de validação da versão portuguesa do McKnight Risk Factor Survey IV. Rev Iberoam Diagn Ev 32:143-170

26. Thompson MA, Gray J (1995) Development and validation of a new body-image assessment scale. J Pers Assess 64:258-269

27. Francisco R, Narciso I, Alarcão M (2012) (In)Satisfação com a imagem corporal em adolescentes e adultos portugueses: Contributo para o processo de validação da Contour Drawing Rating Scale. Rev Iberoam Diagn Ev 34:61-88

28. Fairburn C, Beglin S (1994) Assessment of eating disorders: interview or self-report questionnaire? Int $\mathrm{J}$ Eat Disord 16:363-370

29. Machado P, Martins C (2010) Eating Disorder Examination Questionnaire (EDE-Q): Psychometric properties and norms for the Portuguese population (in preparation)

30. Anderson J, Gerbing D (1988) Structural equation modeling in practice: a review and recommended two-step approach. Psychol Bull 103:411-423

31. Ricciardelli L, McCabe M (2004) A biopsychosocial model of disordered eating and the pursuit of muscularity in adolescent boys. Psychol Bull 130:179-205

32. Hu L-T, Bentler P (1999) Cutoff criteria for fit indexes in covariance structure analysis: conventional criteria versus new alternatives. Struct Equ Model 6:1-55

33. Hayes A (2009) Beyond Baron and Kenny: statistical mediation analysis in the new millennium. Commun Monogr 76:408-420

34. Kline R (2005) Principles and practice of structural equation modeling. Guilford Press, New York

35. Bentler P, Chou CP (1987) Practical issues in structural modeling. Sociol Method Res 16:78-117

36. Petrie TA (1996) Differences between male and female college lean sport athletes, nonlean sport athletes, and nonathletes on behavioral and psychological indices of eating disorders. J Appl Sport Psychol 8:218-230

37. Armstrong S, Oomen-Early J (2009) Social connectedness, selfesteem, and depression symptomatology among collegiate athletes versus nonathletes. J Am Coll Health 57:521-526

38. Findlay L, Bowker A (2009) The link between competitive sport participation and self-concept in early adolescence: a consideration of gender and sport orientation. J Youth Adolesc 38:29-40

39. Thomas J, Keel P, Heatherton T (2005) Disordered eating attitudes and behaviors in ballet students: examination of environmental and individual risk factors. Int J Eat Disord 38:263-268

40. Francisco R, Alarcão M, Narciso I (2012) Aesthetic sports as high risk contexts for eating disorders: young elite dancers and gymnasts perspectives. Span J Psychol 15:265-274

41. Torstveit M, Rosenvinge J, Sundgot-Borgen J (2008) Prevalence of eating disorders and the predictive power of risk models in female elite athletes: a controlled study. Scand J Med Sci Sport 18:108-118

42. Harter S (1999) The construction of the self: a developmental perspective. Guilford Press, New York 